RESEARCH NOTE

\section{Polymorphism of the Pedictive Antigenic Sites on the V3 Loop of Brazilian HIV-1 Subtype B Strains}

MG Morgado/, ML Guimarães,
CBG Gripp, I Neves Jr, CI Costa, VG
Veloso dos Santos*, MI Linhares-de-
Carvalho**, B Galvão-Castro***,
V Bongertz, HEC/FIOCRUZ AIDS
Clinical Research Group*

Departamento de Imunologia, Instituto Oswaldo Cruz, Av. Brasil 4365, 21045-900 Rio de Janeiro, RJ, Brasil *Hospital Evandro Chagas, Rio de Janeiro, RJ, Brasil **Ambulatório da Previdência da Arquidiocese do Rio de Janeiro, RJ, Brasil ***LASP/CPqGM, FIOCRUZ, Salvador, BA, Brasil

Key words: HIV-1 polymorphism - antigenic sites V3 loop - Brazil

The Human Immunodeficiency Virus Type 1 (HIV-1) isolates have been found to be highly divergent both in nucleotide and amino acid sequences. Phylogenetic analysis of the worldwide distributed isolates showed at least nine HIV-1 subtypes (A-I) included in the majoritary $M$ group, in addition to the highly divergent group $\mathrm{O}$ (G Myers et al. 1994 Human retroviruses and AIDS: A Compilation of Nucleic and Amino Acid Sequences, Los Alamos, Natl Lab Los Alamos, New Mexico, W Janssens et al. 1994 AIDS Res Hum Retrov 10: 877-879, LG Kostrikis et al. 1995 $J$ Virol 69: 6122-6130). This striking genetic variability of HIV-1 isolates, specially at the immuno-

Supported by grants from the UNDP/World Bank/Brazilian Ministry of Health-STD-AIDS Program, GPA/ WHO, Programa Institucional de AIDS/FIOCRUZ and Conselho Nacional de Desenvolvimento Científico e Tecnológico - CNPq.

*HEC/FIOCRUZ AIDS Clinical Research Group: B Grinszteijn, MCG Galhardo, MRC Guimarães, VC Rolla, S Cavalcante

${ }^{+}$Corresponding author. Fax: 55- 21-280.1589

Received 7 December 1995

Accepted 10 January 1996 logically important regions of the envelope protein gp120, can be limiting for the development of a broadly protective vaccine. Indeed, one of the important antigenic epitopes recognized by neutralizing antibodies, is the type-specific principal neutralizing determinant (PND) which maps in the top of the V3 loop (K Javaherian et al. 1989 Proc Natl Acad Sci USA 86: 6768-6772, K Javaherian et al. 1990 Science 250: 1590-1593, GD La Rosa et al. 1990 Science 249: 933-935).

Moreover, antigenic epitopes stimulating cell mediated immune responses have also been mapped within this highly variable region (B Warren et al. 1989 AIDS 4: 448-456, Clerici et al. 1991 J Immunol 146: 2214-2219, AM Geretti et al. 1994 Scan J Immunol 39: 355-362).

At least three HIV-1 subtypes, B, F and C have been found in Brazil (KE Potts et al. 1993 AIDS 7: 1191-1197, MG Morgado et al. 1994 AIDS Res Hum Retrov 10: 569-576, J Lowagie et al. 1994 AIDS Res Hum Retrov 10: 561-567, WHO Network for HIV Isolation and Characterization 1994 AIDS Res Hum Retrov 10: 1327-1343). Moreover, an HIV-1 recombinant B/F genome has also been identified (EC Sabino et al. 1994 J Virol 68: 63406346). However, there is a clear predominance up to now of isolates belonging to the B subtype. This subtype is also highly prevalent in the USA and Europe, and the majority of the molecules candidate to the development of an anti-HIV/AIDS vaccine were obtained from LAI, MN, or SF-2 HIV-1 isolates, all of them included in this subtype. Moreover, a high percentage of Brazilian subtype B isolates present a typical amino acid composition at the conserved crown of the gp 120 V3 loop, with the GWGR tetrapeptide replacing the conserved GPGR, in addition to other amino acid substitution flanking this region.

Brazil is one of the four countries selected by the World Health Organization as possible vaccine trial field sites. Nevertheless, as more sequence data from Brazilian HIV-1 subtype B isolates became available it is possible to perform a more accurate analysis of such diversity and to evaluate its importance on the specificity of the immune response for future anti-HIV/AIDS vaccine candidates. Up to now, nucleotide sequences from 71 Brazilian HIV-1 samples belonging to subtype B have been published by different groups. The comparative analysis of the deduced amino acid sequences of the gp $120 \mathrm{C} 2-\mathrm{V} 3 \mathrm{re}-$ gion of those samples permitted the identification of the two main groups of subtype B samples circulating in Brazil (Fig. 1). Indeed, the conserved GPG motif in the tip of the V3 loop, simi- 
v LOOP

Fig.1: alignment of the deduced amino acid sequences of the gp $120 \mathrm{C} 2-\mathrm{V} 3$ regions from the two Brazilian subtype B consensus sequences. The consensus sequences were derived based on the alignment of 71 published sequences classified as subtype B (KE Potts et al. 1993 AIDS 7: 1191-1197, MG Morgado et al. 1994 AIDS Res Hum Rretrov 10: 569-576, J Lowagie et al. 1994 AIDS Res Hum Retrov 10: 561-567, WHO Network for HIV Isolation and Characterization Report 1994 AIDS Res Hum Retrov 10: 1327-1343, E Sabino et al. 1994 J Virol 68: 6340-6346, JC Couto-Fernandez et al. 1994 AIDS Res Hum Retrov 10: 11571163, SM Costa 1995 AIDS Res Hum Retrov 11: 1143-1145).

lar to the classical North American/European subtype B strains, was identified in $46.5 \%$ of the Brazilian HIV-1 samples, whereas $39.4 \%$ beared the motif GWG, typically identified among Brazilian strains. The remaining $14.1 \%$ presented other amino acid sequences at this region (GFG, GLG, APG, etc). This $\mathrm{P} \rightarrow \mathrm{W}$ modification was consistently associated to other amino acid substitutions and the predicted consensus sequences for both groups differed in 10 of the 100 amino acid positions analyzed. Moreover, the GPGQ motif at the crown of the V3 loop was detected in only one out of 71 Brazilian subtype B samples analyzed, while this motif is typically seen in subtypes A, C, D and E strains (Myers et al. loc. cit.) and was recently found to be highly frequent among subtype B samples from Thailand (ML Kalish et al. 1995 AIDS 9: 851-857).

Low homology at the V3 loop was detected when the Brazilian subtype B samples were aligned with some North American/European HIV-1 strains as LAI, MN and SF-2, which are under

\section{TABLE I}

Percent V3 loop homology between 71 Brazilian HIV-1 samples identified as subtype B and the North American/ European strains used for vaccine development

\begin{tabular}{ccc}
\hline \multirow{2}{*}{$\begin{array}{c}\text { HIV-1 } \\
\text { strains }\end{array}$} & \multicolumn{2}{c}{ Homology with } \\
\cline { 2 - 3 } & $\begin{array}{c}\text { Brazilian HIV-1 } \\
\text { subtype B }\end{array}$ & $\begin{array}{c}\text { Non-Brazilian } \\
\text { subtype B } \\
\text { (mean) }\end{array}$ \\
\hline LAI & $58 \%-78 \%$ & $75 \%$ \\
MN & $64 \%-83 \%$ & $83 \%$ \\
SF-2 & $71 \%-91 \%$ & $89 \%$ \\
\hline
\end{tabular}

$\bar{a}$ : based on G Myers et al. (1994 Human Retroviruses and AIDS: A Compilation of Nucleic and Amino Acid Sequences, Los Alamos National Laboratory, Los Alamos, NM, USA). evaluation for anti-HIV/AIDS vaccine development (Table I).

In order to evaluate the impact of those amino acid modifications on the secondary structure and antigenicity of this region, a computer assisted analysis was conducted using Peptide Structure in GCG sequence analysis software package (version 7). The prediction of the secondary structure was based on the Chou-Fasman method (1978 $A d v$ Enzymol 47: 45-158) and the Jameson-Wolf algorithm was used to evaluate antigenic sites based on the hydrophilicity, flexibility, surface probability and secondary structure parameters. Fig. 2 shows the predictive structures of the gp $120 \mathrm{C} 2-$ V3 region of the two Brazilian HIV-1 subtype B consensus sequences (GPG and GWG), compared to the corresponding regions on the $\mathrm{MN}$ and $\mathrm{LAI}$ HIV-1 isolates.

Differences in the secondary structure of the corresponding peptide chains, as well as in the glycosilation sites and in the localization and antigenic index of the antigenic sites could be detected between the samples analyzed. It has been shown that the GPGR residues at the tip of the V3 loop form antigenic beta-turns ( $\mathrm{J}$ Ghaira et al. 1994 Science 264: 82-85), however, this structure is lost in the sample presenting the GWGR motif at this region (this paper). This low probability for beta-turn conformation in this region was also described for other divergent V3 loop residues as GPWG, GQGQ or GLGQ, which were associated to a lower antigenicity (GA Pestano et al. 1995 AIDS Res Hum Retrov 11: 589-596). Indeed, the reactivity of sera from Brazilian HIV-1 infected individuals against peptides corresponding to the crown of the V3 loop was lower than it has been shown for sera from North American/European HIV-1 positive individuals (EW Carrow et al. 1991 AIDS Res Hum Retrov 7: 831838, V Bongertz et al. 1994 Braz J Med Biol Res 27: 1225-1236). Moreover, low crossreactivity 


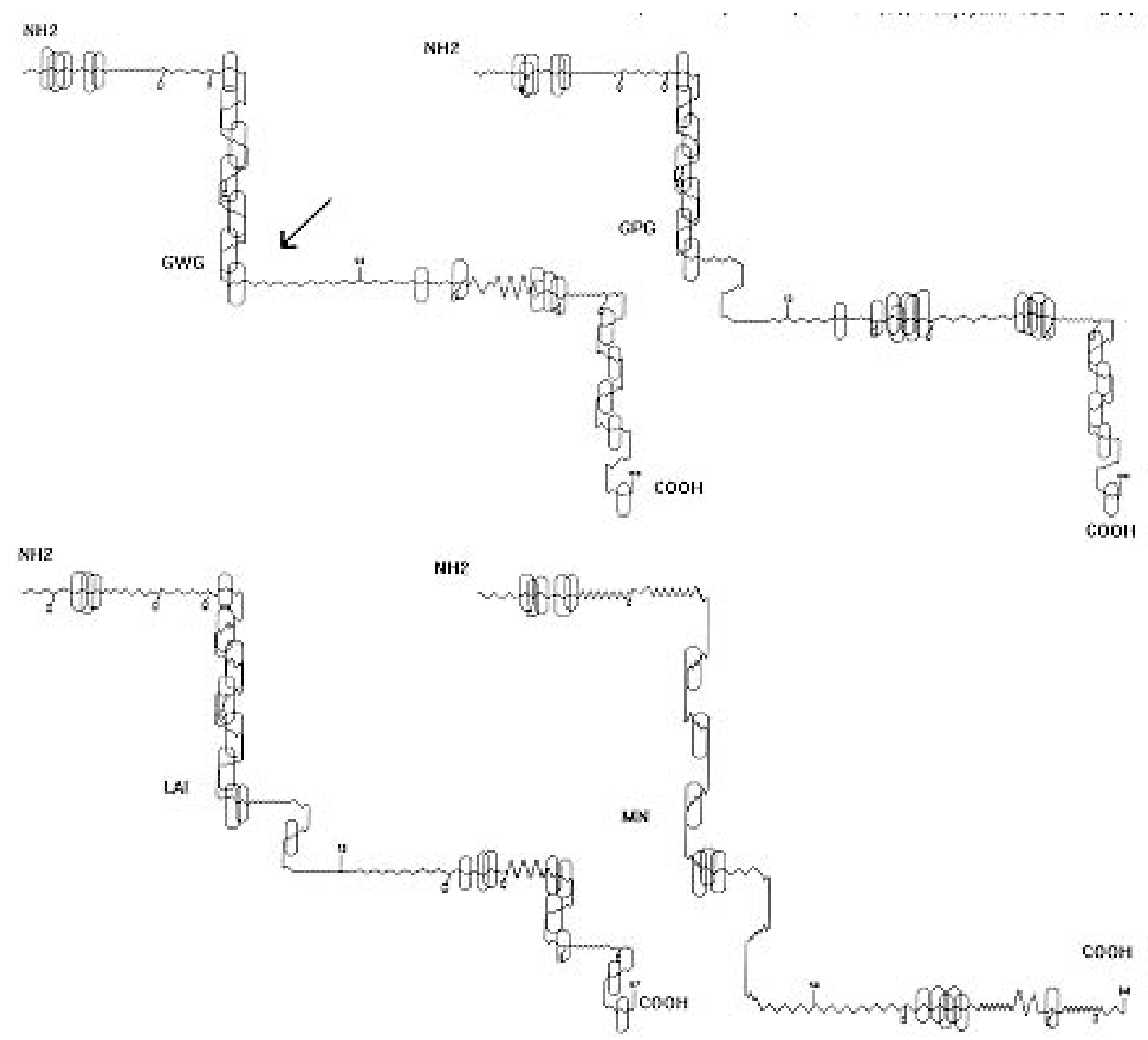

Fig.2: predicted structures of the gp $120 \mathrm{C} 2$-V3 regions from the two subtype B Brazilian consensus sequences (GPG and GWG) and the MN and LAI HIV-1 isolates. Dull saw-tooth waves predicted random coils, turns, beta-turn regions, large waves, alphahelix and shark-tooth waves, beta-sheet structure. Glycosilation sites $(\sigma)$ and antigenic sites $(0)$ are indicated.

with peptides containing the IGPGR and MGWGR was detected among sera from HIV-1 positive individuals infected respectively with the corresponding viral sequences (R CheingsongPopov et al. 1994 AIDS Res Hum Retrov 10: 13791386, C-P Pau et al. 1994 AIDS Res Hum Retrov 10: 1369-1377). However, such restricted antibody response was not detected when functional neutralizing antibodies against viruses containing those sequences were analyzed (Bongertz et al. Mem Inst Oswaldo Cruz, this issue).

In order to assess whether the polymorphism in this region could also restrict $\mathrm{T}$ cell antigenic response, we are currently evaluating the lymphoproliferative response of HIV-1 positive individuals infected in Brazil, against synthetic peptides corresponding to those polymorphic sequences. Briefly, triplicates of PBMC from HIV-1positive individuals were cultivated at $2 \times 10^{6}$ cells/ml in RPMI-1640 (Sigma Chem Co., USA) suplemented with $10 \%$ of heat-inactivated human AB serum (Sigma Chem Co., MO, USA) for 5-6 days in the presence of $10-20 \mu \mathrm{g} / \mathrm{ml}$ each peptide (Chyron Mimotopes, $\mathrm{Au}$ ), or PHA mitogen (Sigma Chem Co., USA) at $5 \mu \mathrm{g} / \mathrm{ml}$. One $\mathrm{uCi}$ of $[3 \mathrm{H}]$ methylthymidine (Amersham, UK) with a specific activity of 20Ci./mmol was added to each well 16-18 hr before harversting. Preliminary data with 10 asymptomatic HIV-1 positive individuals are presented in Table II. PBMC from five out of 10 individuals analyzed did not react with the GWG peptide while reacting with one or 


\section{TABLE II}

Lymphoproliferative response ${ }^{a}$ of Brazilian HIV-1 positive individuals to peptides corresponding to the tip of the V3 loop of MN, LAI and SF-2 HIV-1 isolates, as well as from the two HIV-1 subtype B consensus sequences typically found in Brazil (GPG and GWG)

\begin{tabular}{cccccc}
\hline \multirow{2}{*}{ Patients } & \multicolumn{5}{c}{ Synthetic peptides } \\
\cline { 2 - 6 } & $\mathrm{MN}^{b}$ & LAI $^{c}$ & SF-2 $^{d}$ & GPG $^{e}$ & GWG $^{f}$ \\
\hline 01 & - & +++ & ND & ND & +++ \\
02 & ++ & - & ND & ND & + \\
03 & + & - & - & - & + \\
04 & - & - & - & + & - \\
05 & + & + & + & + & - \\
06 & - & + & ++ & + & + \\
07 & - & - & + & + & - \\
08 & - & - & ND & ND & + \\
09 & +++ & - & - & ++ & - \\
10 & - & + & - & + & - \\
\hline
\end{tabular}

$a$ : the data were scored in terms of the stimulation index (experimental counts/counts without peptide or mitogenic stimulation). Synthetic peptides used were:

$b$ : RHIGPGRAFYTTKN; $c$ : RIQRGPGRAFVTIGK $d$ : SIYIGPGRAFHTTGR; $e$ : SIHIGPGRAFYTTGD $f$ : SIHMGWGRAFYATGE. Negative samples had scores $<2.0$. Positive samples were scored as $+>2.0$ $<5.0,++>5.0<10.0$ and $+++>10.0$. ND: not done more peptide containing the GPG motif. Only one patient showed lymphoproliferative response exclusively to the GWG peptide, although with a quite low stimulation index. The other four individuals, who were also reactive to this peptide, showed lymphoproliferative response to at least one other peptide containing the GPG motif at the crown of the V3 loop. The stimulation indexes were, however, highly variable among them. All these individuals were infected with HIV-1 subtype B isolates, determined by Heteroduplex Mobility assay as described (EL Delwart et al. 1993 Science 262: 1257-1261), and nucleotide sequencing is being carried out to discriminate the V3 loop variations inside the subtype B.

Despite interisolate peptide sequence heterogeneity, our data showed that HIV-1 positive individuals reactive to the typical Brazilian HIV-1 B peptide (GWG) had broad lymphoproliferative reaction with other peptides such as the MN, LAI, SF-2 or B consensus (GPG), suggesting conservative $T$ cell epitopes in the region covered by those peptides. Further studies will be necessary to assess this question in order to give support for future anti-HIV/AIDS immunoprophylactic trials in Brazil. 